\title{
Comportamientos sexuales en mayores de 18 años con diagnóstico VIH/SIDA en tres ciudades de Colombia 2011
}

\section{Sexual behaviors in over 18 years old with a diagnosis of HIV/AIDS in three cities of Colombia 2011}

\section{Comportamentos sexuais em mais de 18 anos com diagnóstico de HIV / AIDS em três cidades na Colômbia 2011}

\author{
Adriana del Pilar Arcila-Rivera MD., Esp., MSc. * \\ Luis Ángel Toro-López. ** \\ Juan David Cañaveral-Orozco. ** \\ Dayanne González-Lozano. **
}

\author{
Leidy Diana Henao-González. ** \\ Luberth Carmén-Dussán, Ing., Esp., MSc. *** \\ Mauricio Castaño-Ramírez, MD., Esp. ****
}

\begin{abstract}
Resumen
Objetivo: Describir los comportamientos sexuales en personas VIH positivas en tres ciudades de Colombia antes y después de conocer el diagnóstico e identificar cambios en estos comportamientos. Metodología: Estudio descriptivo, de corte trasversal, en el que participaron 85 individuos de tres ciudades colombianas, entrevistados durante el año 2011. Se cumplió con los parámetros éticos para investigaciones con personas. Para el análisis estadístico se utilizó el software SPSS 18.0. Resultados: Se encontraron diferencias significativas al comparar el comportamiento sexual antes y después de conocer el diagnóstico $\mathrm{VIH}$ positivo. el $23 \%$ de los entrevistados manifestó no haber iniciado actividades sexuales después del diagnóstico, el $62.5 \%$ empezó a usar el condón en todas sus relaciones sexuales. Las relaciones sexuales con personas VIH positivo aumentaron a un $35.4 \%$. Las infecciones de transmisión sexual disminuyeron a un $12.3 \%$. El consumo de alcohol previo al inicio de relaciones sexuales disminuyó a un 31.8\%. Conclusiones: Se encontraron cambios en los comportamientos de riesgo de las personas después de
\end{abstract}

recibir el diagnóstico; sin embargo, dichas modificaciones no son suficientes por tratarse de la trasmisión de una infección mortal y de elevados costos socioeconómicos.[Arcila-Rivera, AP, Toro-López, LA, Cañaveral-Orozco, JD, GonzálezLozano, D, Henao-González, LD, Carmén-Dussan, L, Castaño-Ramírez, M. Comportamientos sexuales en mayores de 18 años con diagnóstico VIH/SIDA en tres ciudades de Colombia 2011. MedUNAB 2016; 19 (2): 95-102]

Palabras clave: VIH; Síndrome de Inmunodeficiencia Adquirida; Consejo Sexual; Conducta Sexual; Infecciones por VIH; Seroprevalencia de VIH; Sexo Seguro; Sexo Inseguro; Parejas Sexuales; Coito.

\begin{abstract}
Objective: To describe sexual behaviors of HIV positive patients in three cities of Colombia, before and after learning diagnosis and to identify changes. Methodology: Quantitative, descriptive, cross-sectional study, with participation of 85 individuals from three cities of Colombia who were interviewed
\end{abstract}

Médica y Cirujana, Especialista en Educación Sexual, Magister en Filosofía. Docente del Departamento de Salud Mental y Comportamiento Humano, Universidad de Caldas, Colombia.

** Médico Interno, Universidad de Caldas, Colombia

*** Ingeniero Químico, Especialista en Estadística, Magister en enseñanza de Matemáticas, Docente Departamental de Matemáticas, Universidad de Caldas Colombia.

**** Médico Psiquiatra Docente del Departamento de Salud Mental y Comportamiento Humano, Universidad de Caldas, Colombia

Autor de correspondencia: Adriana del Pilar Arcila-Rivera, dirección de correspondencia: carrera $9^{\mathrm{a}} \mathrm{N}^{\circ} 68^{\mathrm{a}}-50$, Manizales, Caldas, Colombia. Correo electrónico: adriana.arcila@ucaldas.edu.co.

Artículo recibido: 13 de marzo de 2015 Artículo aceptado: 24 de octubre de 2016 
during 2011. Ethical standards for research involving individuals were met during the study. The SPSS software version 18.0 was used for statistical analysis. Results: Significant differences were found when comparing their sexual behavior before and after knowing HIV positive diagnosis, $23.0 \%$ of respondents said that they had not resumed sexual activity, $62.5 \%$ of the patients began to use the condom in all sexual relations. Sex with HIV-positive people increased a $35.4 \%$. Sexually transmitted infections were reduced to a $12.3 \%$. The consumption of alcohol prior to the onset of sexual intercourse decreased to $31.8 \%$. Conclusions: Changes were found regarding risky behaviors of people after knowing the diagnosis; however, such modifications are not enough regarding the transmission of a deadly infection and with high social and economic costs. [Arcila-Rivera, AP, Toro-López, LA, CañaveralOrozco, JD, González-Lozano, D, Henao-González, LD, Carmén-Dussan, L, Ramírez-Castaño, M. Sexual behaviors in over 18 years old with a diagnosis of HIVIAIDS in three cities of Colombia 2011. MedUNAB 2016; 19 (2): 95-102]

Keywords: HIV; Acquired Immunodeficiency Syndrome; Sex Counseling; Sexual Behavior; HIV Infection; HIV Seroprevalence; Safe Sex; Unsafe Sex; Sexual Partners; Coitus.

\section{Resumo}

Objetivo: Descrever o comportamento sexual em pessoas HIV-positivas em três cidades na Colômbia antes e depois de

\section{Introducción}

En el año 1981, comenzó oficialmente la epidemia del Síndrome de Inmunodeficiencia Adquirida (SIDA) con el reporte del Centro de Control y Prevención de Enfermedades de los Estados Unidos (CDC) de cinco pacientes con neumonía por Pneumocystis carinii (1). Según el CDC, se estima, que a partir de esta fecha han muerto 39 millones de personas con SIDA y que alrededor de 35 millones de personas viven con el Virus de la Inmunodeficiencia Humana (VIH) alrededor del mundo (2). Las regiones más afectadas por la epidemia son los países del África Subsahariana, con cerca del 70\% de los casos de infección por el VIH y el 73\% de la mortalidad mundial por SIDA, seguidos por otras regiones que están afectadas de manera significativa en las que se encuentra incluida Latino América y el Caribe $(2,3)$.

El Programa Conjunto de las Naciones Unidas para El VIH/SIDA (ONUSIDA), menciona que en el 2013, 1,6 millones de personas vivían con el VIH en América Latina, con una cobertura de tratamiento del $45 \%$ y una reducción de las infecciones por VIH en un $3 \%$ en el periodo 2005 2013 (4, 5), lo cual indica la necesidad de realizar evaluaciones constantes de las estrategias que se utilizan en el control de la propagación de la enfermedad.

En Colombia, el primer caso de SIDA se notificó en 1983 y hasta el año 2011 se habían notificado 83,467 casos de conhecer o diagnóstico e identificar mudanças no esses comportamentos. Metodologia: Estudo descritivo de transversal, em que 85 indivíduos participaram em três cidades colombianas, entrevistado em 2011. Eles se conheceram os padrões éticos para a investigação com as pessoas. Para análise estatística foi utilizado SPSS 18.0 software. Resultados: Diferenças significativas na comparação entre o comportamento sexual foram encontrados antes e depois de aprender sobre o diagnóstico de HIV positivo. 23\% dos entrevistados disseram que a atividade sexual não ter iniciado após o diagnóstico, $62,5 \%$ começaram a usar preservativos em todas as relações sexuais. Sexo com pessoas HIV positivas aumentou para $35,4 \%$. infecções sexualmente transmissíveis diminuiu para $12,3 \%$. O consumo de álcool antes do início do sexo diminuiu para 31,8\%. Conclusões: foram encontradas alterações nos comportamentos de risco de pessoas depois de receber o diagnóstico; No entanto, estas alterações não são suficientes, porque é a transmissão de uma infecção fatal e custos socioeconómicos elevados. [Arcila-Rivera, AP, Toro-López, LA, Cañaveral-Orozco, JD, González-Lozano, D, HenaoGonzález, LD, Carmén-Dussan, L, Ramírez-Castaño, M.Comportamentos sexuais em mais de 18 anos com diagnóstico de HIV / AIDS em três cidades na Colômbia 2011. MedUNAB 2016; 19 (2): 95-102]

Palavras-chave: HIV; Síndrome de Imunodeficiência Adquirida; Aconselhamento Sexual; Comportamento Sexual; Infecções por HIV; Soroprevalência de HIV; Sexo Seguro; Sexo sem Proteção; Parceiros Sexuais; Coito.

VIH/SIDA (6). Durante el año 2011, se diagnosticaron 7,991 casos nuevos, de los cuales 5,830 correspondían a personas infectadas por VIH y 1,551 a personas con SIDA. Además, se notificaron 610 muertes relacionadas con el SIDA (7). El $98 \%$ de la transmisión de la infección se dio por vía sexual y afectó principalmente a las personas con edades entre 25 y 34 años, con un mayor predominio en los hombres (71.93\%) (7).

En el departamento de Caldas hasta el 2010 se tenían 1,188 casos notificados; en Risaralda la cifra ascendía a 2,465 y en Quindío a 1,775 casos (8). Para ese año, Quindío presentó la segunda tasa de incidencia del país: 32,7 , mientras que la tasa nacional fue de 16,5 por 100.000 habitantes (8).

En cuanto al género, la prevalencia es mayor en hombres que tienen sexo con hombres (HSH); sin embargo, en los últimos años se ha encontrado un aumento de la población femenina con VIH/SIDA con una relación hombre mujer de 2:1 (9-11). La infección es más prevalente en HSH y en trabajadores sexuales. Además, se han encontrado otras condiciones que generan mayor vulnerabilidad como: la pobreza, el desplazamiento interno, la marginalidad y la desigualdad de género (10).

De otra parte, se han encontrado comportamientos sexuales que favorecen la propagación de la enfermedad que son: el alto intercambio de parejas sexuales, el bajo uso del preservativo, el uso de sustancias psicoactivas y el desconocimiento del estado serológico de la pareja (12-14). 
Algunos países han desarrollado programas encaminados a controlar la propagación de la epidemia mediante estrategias que buscan la aceptación del diagnóstico, la promoción de pautas de autocuidado y la motivación hacia comportamientos sexuales seguros, entre ellas el uso constante del preservativo, la disminución en el consumo de sustancias psicoactivas previo a las actividades sexuales, la disminución del número de parejas sexuales y la notificación del estado serológico (14-16).

En Colombia, aunque el sistema de salud ofrece asesoría a las personas que se encuentran infectadas, no se conoce su eficacia, así como la de otras estrategias dirigidas al control de la epidemia, y no se tiene información acerca de los comportamientos sexuales de las personas infectadas, ni tampoco los cambios que existen en estos una vez conocido el diagnóstico. Por ello, y teniendo en cuenta que la práctica de una sexualidad responsable es una conducta eficaz para el control de la epidemia es necesario investigar al respecto.

El objetivo del presente estudio fue describir los comportamientos sexuales en personas con VIH/SIDA del eje cafetero antes y después de conocer el diagnóstico e identificar cambios en estos comportamientos.

\section{Metodología}

Este es un estudio descriptivo, de corte trasversal, aprobado por el Comité de Ética de la Facultad de Ciencias para la Salud de la Universidad de Caldas y por los coordinadores de las Instituciones Prestadoras de Salud (IPS). Se utilizó la técnica de muestreo por conveniencia entendido como el método no probabilístico de seleccionar sujetos que están disponibles; entre los criterios de inclusión estuvo la aceptación solo de personas con diagnóstico de VIH/SIDA mayores de 18 años que asistían a controles por consulta externa de la Fundación Girasol de Pereira y el GRUPO VIHDA IPS del eje Cafetero (Armenia - Pereira Manizales), diagnosticados en un periodo de tiempo superior a un año y que aceptaran participar en la investigación; como criterio de exclusión se señaló personas con deterioro cognitivo secundario o en fase terminal de su enfermedad que impidieran responder a la entrevista.

Las entrevistas se llevaron a cabo en los servicios de consulta externa de las IPS mencionadas, en los horarios destinados al control médico, todos los pacientes citados a control asistieron, y todos aceptaron participar en el estudio, ninguno de ellos presentaba deterioro cognitivo que impidiera realizar la entrevista.

Se solicitó la firma del consentimiento informado, se les garantizó la confidencialidad, y se siguieron las normas colombianas vigentes para la investigación en salud y la declaración de Helsinki.

La realización de las entrevistas estuvo a cargo de los cuatro médicos internos, quienes recibieron entrenamiento para la ejecución de la entrevista estructurada, en ella se preguntó acerca de variables sociodemográficas y de los comportamientos sexuales antes y después del diagnóstico. El análisis de los datos se efectuó con el software estadístico SPSS versión 18.0; mediante análisis univariado se establecieron las frecuencias de las variables evaluadas; posteriormente, se compararon los comportamientos sexuales antes y después del diagnóstico mediante la prueba de independencia $\chi^{2}$

\section{Resultados}

Se captó una muestra de 85 sujetos con diagnóstico de VIH/SIDA(61 hombres y 24 mujeres), con una media de 39 años (DE 18-71), todos aceptaron participar en la investigación. En su mayoría las personas vivían en condiciones socioeconómicas bajas dentro de la estratificación de las viviendas en Colombia encontrando que: el $37 \%$ pertenecían al estrato $1 ; 32 \%$ al estrato $2 ; 31 \%$ al estrato $3 ;$ y $1 \%$ al estrato 4 . No se encontraron sujetos de estratos 5 y 6 (Tabla 1$)$.

Tabla 1. Características sociodemográficas.

\begin{tabular}{lcc}
\hline \multicolumn{1}{c}{ Característica } & $\mathrm{N}$ & $\%$ \\
\hline Sexo & & \\
\hline Masculino & 61 & 71.8 \\
\hline Femenino & 24 & 28.2 \\
\hline Estado civil & & \\
\hline Soltero/Separado/Viudo & 60 & 70.5 \\
\hline Casado/Unión libre & 25 & 29.5 \\
\hline Estrato Social & & \\
\hline Estrato 1 & 31 & 36.5 \\
\hline Estrato 2 & 27 & 31.8 \\
\hline Estrato 3 & 26 & 30.6 \\
\hline Estrato 4 & 1 & 1.2 \\
\hline Régimen de Salud & & \\
\hline Contributivo & 24 & 28.2 \\
\hline Subsidiado & 61 & 71.8 \\
\hline Escolaridad & & \\
\hline Primaria & 25 & 29.4 \\
\hline Secundaria & 40 & 47.1 \\
\hline Estudios técnicos & 3 & 15.3 \\
\hline Universidad & 4.7 \\
\hline Ninguno & & \\
\hline Ocupación & & \\
\hline Empleado & & \\
\hline Estudiante & & \\
\hline Pensionado & & \\
\hline Desempleado & & \\
\hline & & \\
\hline
\end{tabular}


Con respecto al diagnóstico, se encontró que el promedio de edad en el cual se diagnosticó VIH positivo fue 33 años (IC 95\% 31-35). El 82\% de los participantes recibió asesoría antes y/o después de la prueba diagnóstica, la mitad recibió asesoría previa y posterior a la prueba de laboratorio. El motivo por el cual se realizó la prueba del VIH fue en un $60 \%$ por solicitud médica, $18 \%$ por sospecha de haber estado expuesto a una situación de riesgo, $12 \%$ al conocer que una pareja era VIH positiva y $10 \%$ por curiosidad. En el $96.5 \%$ de los encuestados la posible vía de contagio fue la sexual, el $2.4 \%$ la vía transfusional y el $1.1 \%$ un accidente laboral.

Posteriormente se compararon las prácticas sexuales antes y después del diagnóstico. El 76.5\% de las personas continuó con su vida sexual (Tabla 2). Entre los que continuaron con vida sexual activa no se encontraron diferencias significativas por género, número de parejas sexuales, ni cambios en su orientación sexual. Se excluyeron dos casos, en quienes el número de parejas sexuales correspondía a valores atípicos de 300 y 50 alrededor del tiempo del diagnóstico y que afectaban el promedio de parejas sexuales.
Se encontró una disminución de las prácticas sexuales bajo efectos del alcohol, y la presentación de infecciones de transmisión sexual (ITS). Además, se encontró un aumento en el uso del condón y de las relaciones sexuales con personas VIH positivas (Tabla 3).

Tabla 2. Tiempo tras el cual se reinician las prácticas sexuales después de conocer el diagnóstico.

\begin{tabular}{lcc}
\hline Tiempo & $\mathrm{n}$ & $\%$ \\
\hline Antes de 2 meses & 14 & 16.5 \\
\hline Entre 2 y 6 meses & 16 & 18.8 \\
\hline Entre 7 y 12 meses & 12 & 14.1 \\
\hline Después de 12 meses & 23 & 27.1 \\
\hline No ha reiniciado & 20 & 23.5 \\
\hline Total & 85 & 100 \\
\hline
\end{tabular}

Tabla 3. Prácticas sexuales antes y después del diagnóstico.

\begin{tabular}{|c|c|c|c|c|c|}
\hline \multirow[t]{2}{*}{ COMPORTAMIENTOS SEXUALES } & \multicolumn{2}{|c|}{ Antes del diagnóstico } & \multicolumn{2}{|c|}{$\begin{array}{l}\text { Después del } \\
\text { diagnóstico }\end{array}$} & \multirow[t]{2}{*}{ Valor de $\mathrm{p}^{* *}$} \\
\hline & $\%$ & $\mathbf{N}$ & $\%$ & $\mathbf{N}$ & \\
\hline \multicolumn{6}{|l|}{ Tipo de relaciones sexuales } \\
\hline Vaginales & 70 & 60 & 67.7 & 44 & 0.70 \\
\hline Anales & 52.9 & 45 & 47.7 & 31 & 0.52 \\
\hline Orales & 60.0 & 51 & 49.2 & 32 & 0.18 \\
\hline \multicolumn{6}{|l|}{ Uso del condón } \\
\hline Siempre & 7.1 & 6 & 62.5 & 40 & $<0.01$ \\
\hline Algunas veces & 44.7 & 38 & 29.7 & 19 & 0.062 \\
\hline Nunca & 48.0 & 41 & 7.8 & 5 & $<0.01$ \\
\hline \multicolumn{6}{|l|}{ Relaciones sexuales con personas } \\
\hline VIH positivo & 9.4 & 8 & 35.4 & 23 & $<0.01$ \\
\hline Presencia de ITS* & 27.1 & 23 & 12.3 & 8 & 0.02 \\
\hline $\begin{array}{l}\text { Actividades sexuales bajo efecto de } \\
\text { alcohol }\end{array}$ & 58.8 & 50 & 31.8 & 27 & 0.04 \\
\hline $\begin{array}{l}\text { Actividades sexuales bajo efecto de } \\
\text { sustancias psicoactivas }\end{array}$ & 15.3 & 13 & 5.9 & 5 & 0.04 \\
\hline
\end{tabular}


Entre los que comunicaron el diagnóstico VIH/SIDA (Tabla 4), el $67.0 \%$ fueron mujeres, el $71.4 \%$ informó tener una única pareja en los últimos 6 meses y el $64.3 \%$ de ellos manifiestan usar siempre el condón. Respecto a la edad y la comunicación del diagnóstico se establecieron dos rangos: los menores e iguales a 30 años y de 31 años en adelante. Se encontró que la muestra con edad igual o menor a 30 años comunica con mayor frecuencia el diagnóstico con una diferencia estadística significativa, $(\mathrm{p}$ valor para $\left.\chi^{2} 0.048\right)$.

El $88.2 \%$ de las personas encuestadas se encontraban en tratamiento. De las personas que no continuaron el tratamiento, el $66.7 \%$ lo atribuyeron a fallas en la entrega de medicamentos por parte de su EPS y los restantes refieren motivos personales para no hacerlo. El $84.7 \%$ afirma que la atención que le presta el personal de salud es excelente, el $11.8 \%$ manifiesta que es aceptable y el $3.5 \%$ que es deficiente.

\section{Discusión}

La investigación muestra que existen cambios en el comportamiento sexual, que se manifiesta por un incremento en el uso del condón, en la disminución de las ITS, disminución de las relaciones sexuales bajo efecto del alcohol y un mayor número de relaciones sexuales entre personas que conocen su estado serológico VIH. Es probable que las asesorías fueran un factor importante para la adopción de estos cambios, aunque también podrían ser resultado del impacto de la experiencia negativa que resulta de recibir este diagnóstico, o del deterioro del estado de salud lo cual los lleva a modificar sus comportamientos. A pesar de que uno de cada cinco se convierte en abstinente sexual, similar a lo encontrado en el estudio de estigma y discriminación en Colombia (17), el promedio de parejas sexuales no disminuye, aspecto que debe ser tenido en cuenta para trabajarse desde los programas de control de la epidemia.

Las personas que asisten a las fundaciones donde se realizaron las encuestas pertenecen en su gran mayoría al régimen subsidiado y en menor proporción al régimen contributivo, esto puede explicar por qué no se encontraron personas de estratos altos. Es probable que las personas de estratos altos tengan mayores posibilidades de acceso a servicios complementarios y más conocimiento de sus derechos en salud, por lo cual no requieran los servicios adicionales ofrecidos por el sistema de salud.

Al igual que el estudio de Estigma y Discriminación (17), se encontró que la mitad de los entrevistados recibieron asesoría antes y después de la prueba diagnóstica. Sin embargo, se encuentra una diferencia en cuanto a las personas que no recibieron asesoría, mientras que a nivel nacional cerca de la cuarta parte no recibieron asesoría, en el eje cafetero se reduce a un $12.0 \%$; lo anterior puede ser explicado por la falta de uniformidad en la aplicación de guías y protocolos, por diferencias en las muestras seleccionadas, o por factores no conocidos que merecen ser investigados.

Llama la atención que la realización de la prueba para VIH en un número importante de casos fue por solicitud del médico, sin embargo, no existe claridad si esta solicitud fue motivada por la presencia de síntomas que sugieran el diagnóstico de SIDA o como parte de programas de

Tabla 4. Comunicación del diagnóstico.

\begin{tabular}{llccc}
\hline Comunicación del diagnóstico & Categoría & n & Frecuencia (\%) & IC 95\% \\
\hline \multirow{3}{*}{ Ex parejas sexuales } & A algunas & 33 & 38.5 & $(0.26 ; 0.51)$ \\
& A ninguna & 39 & 46.2 & $(0.33 ; 0.58)$ \\
& A todas & 13 & 15.3 & $(0.076 ; 0.264)$ \\
Parejas sexuales actuales & A algunas & 23 & 27.7 & $(0.173 ; 0.401)$ \\
& Nunca & 25 & 29.2 & $(0.186 ; 0.418)$ \\
& Siempre & 37 & 43.1 & $(0.308 ; 0.559)$ \\
Familiares y amigos & Algunas veces & 44 & 52.3 & $(0.395 ; 0.648)$ \\
& Nunca & 11 & 12.3 & $(0.054 ; 0.228)$ \\
& Siempre & 30 & 35.4 & $(0.239 ; 0.482)$ \\
Personal de salud & Algunas veces & 8 & 9.2 & $(0.034 ; 0.190)$ \\
& Nunca & 8 & 9.2 & $(0.034 ; 0.190)$ \\
& Siempre & 69 & 81.5 & $(0.699 ; 0.900)$ \\
\hline
\end{tabular}


detección precoz. Por lo tanto, es necesario conocer en qué momento se están solicitando las pruebas serológicas con el fin de determinar si se está realizando un reconocimiento precoz de la infección y facilitar el control de la epidemia.

En la presente investigación se encontró que al año de recibir el diagnóstico, poco más de la mitad de las personas entrevistadas habían reiniciado la vida sexual y una cuarta parte más la reinició después del año, mientras que un $23.0 \%$ manifestó no tener vida sexual activa, cifras similares a las obtenidas por el Estudio de Estigma y Discriminación el cual reveló que el $82.0 \%$ de los entrevistados son sexualmente activos y el $18.0 \%$ no lo son (17). Entre las explicaciones que pueden existir para los cambios en la actividad sexual están: el impacto que genera la enfermedad, factores biológicos que favorezcan una disminución de la libido, cambios actitudinales con respecto a la sexualidad o distintas variables que no han sido exploradas. Los cambios en la conducta sexual, como el uso de condón, evitar relaciones sexuales bajo efecto del alcohol y disminución del número de parejas sexuales se encuentran asociadas a la disminución de la transmisión de ITS. Aunque este es un estudio transversal, los resultados indican que una sexualidad responsable genera un impacto positivo con la disminución de la transmisión de otras ITS entre los que continuaron con su vida sexual activa.

Aunque la adopción de prácticas de sexo seguro por parte de las personas diagnosticadas con el VIH es innegable, no se puede desconocer que existe todavía un grupo importante de personas que mantiene comportamientos de riesgo para sí mismos y la comunidad. Similar a otros estudios, las personas tienen dificultad para comunicar el diagnóstico a sus parejas (18), existen personas que continúan presentando ITS, y otras prácticas sexuales de riesgo para la propagación de la epidemia $(19,20)$.

Con respecto a la comunicación del diagnóstico, cerca de la mitad de los entrevistados informa el diagnóstico a las parejas sexuales actuales. En la literatura internacional se encuentran datos dispares, los porcentajes de comunicación del diagnóstico van desde muy bajos en ciudades como San Francisco en un $40.0 \%$ aproximadamente, hasta cifras elevadas en Brasil en un 93.0\% (18, 21-23). Estas variaciones dependen de la preferencia sexual de los pacientes y del género. En general, se ha observado que las mujeres son quienes en mayor proporción hacen esta revelación, lo cual es consistente con los hallazgos de este estudio (18). Así mismo, se observa una mayor comunicación entre personas con orientación heterosexual que entre aquellos con orientación bisexual u homosexual (18). Estas diferencias también se pueden explicar por otros factores como la educación, la cultura y el grado de estigmatización en las diferentes regiones. La Red Colombiana de Pacientes Viviendo con el VIH/SIDA (Recolvih) da a conocer que en el país, al año 2010, el 50.8\% de las personas con VIH revelaban el diagnóstico a sus parejas sexuales (17), cifra similar a la encontrada en el presente estudio. Aunque la comunicación del estado serológico no es garantía para adoptar prácticas de sexo seguro $(22,24)$, esta variable debe ser evaluada con mayor profundidad, teniendo en cuenta que la comunicación sí puede facilitar la práctica de una sexualidad responsable.

Respecto al uso del preservativo en personas contagiadas con el VIH, una meta es alcanzar cifras cercanas al 100\% para lo cual se requieren estrategias adicionales, puesto que el uso adecuado del preservativo ha probado ser una de las medidas más eficaces para la prevención de esta infección (13).

A pesar de la disminución del uso de alcohol y sustancias psicoactivas previo a las relaciones sexuales, sigue siendo alto el número de personas que continúan con esta práctica, resultado que es comparable con lo reportado en estudios realizados en Nueva York y California en los cuales entre una cuarta parte y un tercio de los participantes consumieron alcohol o alguna droga psicoactiva en sus últimas relaciones sexuales $(25,26)$. Reconociendo que este es un comportamiento de alto riesgo para contraer ITS e incluso para la reinfección con el VIH, preocupa la persistencia de esta conducta aún después del diagnóstico y se hace necesario un trabajo riguroso en este campo.

Las actividades de acompañamiento y refuerzo en prácticas de sexo seguro y responsable deben ser mantenidas en el tiempo, algunos estudios señalan que las personas dejan de cuidarse al pasar algunos meses o incluso llegan a pensar que con las terapias antirretrovirales ya no tienen que protegerse $(27,28)$. También es necesario involucrar a estas personas de manera activa en el control de la epidemia, así la persona infectada con el virus debe tener conocimiento claro de que exponer a otros al riesgo de contraer el virus en Colombia constituye el delito de propagación de epidemia $(29,30)$.

\section{Conclusiones}

Se encontraron cambios en los comportamientos de riesgo de las personas después de recibir el diagnóstico de VIH, tales como incremento en el uso del condón, presentación de abstinencia sexual y disminución del uso de alcohol antes de tener las relaciones sexuales.

La disminución de las ITS después del diagnóstico es un indicador importante de la adopción de prácticas de sexo seguro; sin embargo, persiste un $12 \%$ de personas que contraen ITS, lo cual merece atención por parte de las autoridades sanitarias e implementación de más estrategias de prevención. Otro factor que puede favorecer la propagación de la enfermedad es el bajo porcentaje de comunicación del diagnóstico a la pareja entre personas del mismo sexo.

Entre las limitaciones que tiene la investigación se encuentra el tamaño de la muestra, este puede restar poder a 
algunas asociaciones, las dificultades que genera el estigma al cual están sometidos los pacientes y problemas para acceder a los servicios de salud hicieron difícil la captación de un mayor número de participantes en el estudio.

La investigación sugiere que es conveniente explorar las razones por las cuales algunas personas persisten en comportamientos sexuales inseguros después de conocer el diagnóstico y de tener asesoría; es conveniente evaluar la calidad de las asesorías, y generar estrategias de prevención más eficaces con el fin de disminuir la propagación de la enfermedad.

\section{Agradecimientos}

A la Fundación Girasol de Pereira y al GRUPO VIHDA IPS del eje Cafetero quienes, de manera generosa, ofrecieron el apoyo necesario para hacer la investigación a través de la motivación a sus afiliados y el préstamo de sus instalaciones en Manizales, Pereira y Armenia para realizar las entrevistas con los participantes; igualmente, a cada uno de los participantes por sus invaluables aportes.

\section{Referencias}

1. Centers for Disease Control and Prevention. MMWR. Thirty Years of HIV 1981-2011; Jun, 2011; 60 (21): 68993.

2. Centers for Disease Control and Prevention. (Sede Web). Atlanta. 2015. [fecha de acceso 15 de mayo de 2015. URL disponible en: http://www.cdc.gov/hiv/ statistics/basics.html

3. Sepkowitz KA. AIDS the first 20 years. $\mathrm{N}$ Engl J Med. 2001; Jun, 2001; 344(23): 1764-72.

4. ONUSIDA. Informe de ONUSIDA 2014. La epidemia mundial de sida: datos clave mundiales. Disponible en URL: http://www.unaids.org/sites/default/files/ media_asset/20140716_FactSheet_es_0.pdf

5. Caycedo P. Medina R. Hoy hace treinta años apareció la epidemia de VIH/SIDA en el mundo. [Boletín de prensa No 14 de 2011]. Instituto Nacional de Salud, Oficina de comunicaciones. Bogotá, Colombia; Diciembre 1, 2011.

6. Ministerio de Salud y Protección Social. Resumen de situación de la epidemia por VIH/SIDA en Colombia 1983A2011. Bogotá, Colombia 2012.

7. ONUSIDA. Infección por VIH/SIDA en Colombia. Estado del arte. 2002 - 2005. Ministerio de la Protección Social de Colombia, Dirección General de Salud Pública, 2006.

8. UNESCO/ONUSIDA. Un Enfoque Cultural de la Prevención y la Atención del VIH/SIDA en México y Centroamérica. Estudios e Informes - Serie Especial, No. 21. UNESCO, 2004.

9. Hewitt RG, Parsa N, Gugino L. Women's health. The Role of Gender in HIV Progression. AIDS Read. 2001 Jan;11(1):29-33.

10. Sabogal AA. Informe de VIH-SIDA Colombia Periodo XIII Año 2009, SIVIGILA. (En línea). (Fecha de consulta: 10 de febrero de 2011). Disponible en: http://www.ins.gov.co/ lineas-de-accion/Subdireccion-Vigilancia/Informe $\%$ 20de\%20Evento\%20Epidemiolgico/VIH\%202009.pdf

11. Observatorio Nacional de la Gestión en VIH/SIDA. Comportamiento del VIH/SIDA durante el 2010 en Colombia. Ministerio de la Protección Social; Bogotá, agosto de 2011.

12. UNESCO/ONUSIDA, Un Enfoque Cultural de la Prevención y la Atención del VIH/SIDA en México y Centroamérica, Estudios e Informes - Serie Especial, No. 21. UNESCO, 2004.

13. Weller SC, Davis Beaty K. Efectividad del preservativo en la reducción de la transmisión del VIH en heterosexuales. Base de Datos Cochrane de Revisiones Sistemáticas 2002, Issue 1. No.: CD003255. DOI: 10.1002/14651858.CD003255.

14. Fenton KA, Peterman TA. HIV Partner Notification: Taking a New Look. AIDS. 1997; Nov; 11(13):1535-46.

15. Gilson RJC, Mindel A. Sexually transmitted infections. BMJ 2001;322:1160.

16. Van de Perre P. HIV Voluntary Counselling and Testing in Community Health Services. Lancet. 2000; Jul; 356(9224): 86-7.

17. RECOLVIH, IFARMA Y ONUSIDA. Voces positivas: resultados del índice de estigma y discriminación en personas que viven con VIH en Colombia. Bogotá; septiembre 2011.

18. Ciccarone DH, Kanouse DE, Collins RL, Miu A, Chen JL, Morton SC, Stall R. Sex without Disclosure of Positive HIV Serostatus in a US Probability Sample of Persons Receiving Medical Care for HIV Infection. Am J Public Health. 2003; Jun; 93(6): 949-54.

19. Aboim S. Risco e prevenção do HIV/Aids: uma perspectiva biográfica sobre os comportamentos sexuaisem Portugal. Ciênc. Saúdecoletiva. 2012; Jan;17 (1):99-112.

20. Shuper PA, Joharchi N, Irving H, Rehm J. Alcohol as a Correlate of Unprotected Sexual Behavior among People Living with HIVIAIDS: Review and Metaanalysis. AIDS Behav. 2009; Dec; 13(6): 1021-36.

21. Dave SS, Stephenson J, Mercey D E, Panahmand N, Jungmann E. Sexual Behaviour, Condom Use, and Disclosure of HIV Status in HIV Infected Heterosexual Individuals Attending an Inner London HIV Clinic. Sex Transm Infect. 2006; 82:117-120.

22. Paiva V, Segurado A, Ventura EM. Self-disclosure of HIV Diagnosis to Sexual Partners by Heterosexual and Bisexual Men: a Challenge for HIVIAIDS Care and Prevention. Cad. SaúdePública. 2011; Sep; 27(9):1699710.

23. Clarke TR, Gibson R, Barrow G, James S, Abel WD, Barton EN. Disclosure of HIV status among HIV Clinic attendees in Jamaica. West Indian med. j. 2010; Jul; 59(4):445-9.

24. McConnell JJ, Bragg L, Shiboski S, Grant RM. Sexual Seroadaptation: Lessons for Prevention and Sex Research from a Cohort of HIV-positive Men Who Have Sex with Men. PLoS One. 2010; Jan; 5(1): e8831: 1-9

25. Van Devanter N, Duncan A, Burrell-Piggott T, Bleakley A, Birnbaum J, Siegel K et al. The Influence of Substance Use, Social Sexual Environment, Psychosocial Factors, and Partner Characteristics on High-Risk Sexual Behavior among Young Black and Latino Men Who Have 
Sex with Men Living with HIV: A Qualitative Study. AIDS Patient Care and STDs. 2011, 25(2): 113-121.

26. Gorbach PM, Weiss RE, Jeffries R, Javanbakht $M$, Drumright LN, Daar ES et al. Behaviors of Recently HIVInfected Men Who Have Sex with Men in the Year Postdiagnosis: Effects of Drug Use and Partner types. J Acquir Immune DeficSyndr. 2011; Feb; 56(2): 176-182.

27. Juarez VJP, Pozo EJ. Percepciones sobre comportamientos sexuales de riesgo en personas que viven con $\mathrm{VIH} / \mathrm{SIDA}$ y reciben tratamiento antirretroviral en Piura, Perú. Rev. perú. med. exp. salud publica. 2010; May; 27(1):31-7.

28. Crepaz N, Hart TA, Marks G. Highly active antiretroviral therapy and sexual risk behavior: a metaanalytic review. JAMA. 2004 Jul 14; 292(2): 224-236.

29. Congreso de la República de Colombia. Ley 599 de 24 de julio de 2000 por la cual se expide el Código Penal. Diario Oficial No 44.097 de 24 de julio del 2000. Bogotá: Congreso de la República; 2000.

30. Congreso de la República de Colombia. Ley 1220 de 16 de julio de 2008 por la cual se establece el aumento de penas para los delitos contra la Salud Pública. Diario Oficial No. 47.052 de 16 de julio de 2008. Bogotá: Congreso de la República; 2008. 